\title{
ON THE EMBEDDING PROBLEM FOR NONSOLVABLE GALOIS GROUPS OF ALGEBRAIC NUMBER FIELDS: REDUCTION THEOREMS
}

\author{
BY JACK SONN ${ }^{1}$
}

Communicated by H. Bass, January 7, 1972

Let $k$ be a field, $K / k$ a finite Galois extension, $G$ a finite group isomorphic to $\bar{G}=\operatorname{Gal}(K / k), \gamma: \bar{G} \rightarrow G$ an isomorphism and $\Sigma: 1 \rightarrow N \rightarrow{ }_{l} E \rightarrow{ }_{\varepsilon} G \rightarrow 1$ an exact sequence of finite groups. The embedding problem

$$
P=P(K / k, \Sigma, \gamma)
$$

is to construct an extension $L / K$ such that $L / k$ is Galois, and such that there exists an isomorphism $\beta: \bar{E} \rightarrow E$, where $\bar{E}=\operatorname{Gal}(L / k)$, such that $\gamma \cdot \operatorname{Res}_{L / K}=\varepsilon \beta . L$ is called a solution field, $\beta$ a solution isomorphism, and the pair $(L, \beta)$ a solution, to $P$. At times we only require $\beta$ to be monomorphic; in such a context $(L, \beta)$ is called an improper solution, and if $\beta$ is epi, $(L, \beta)$ is a proper solution.

1. Reduction to solvable groups and split extensions. Let $1 \rightarrow N \rightarrow{ }_{l} E$ $\rightarrow_{\varepsilon} G \rightarrow 1$ be an exact sequence of groups, and let $U$ be a subgroup of $E$ such that $U \cdot \imath(N)=E$. Let $E^{*}$ be the semidirect product $(U, N)$, where the action of $U$ on $N$ is given by $n^{u}=l^{-1}\left(u^{-1} \imath(n) u\right)$, for $n \in N, u \in U$. Let the mapping $\eta: E^{*} \rightarrow E$ be defined by $\eta((u, n))=u l(n)$. One verifies easily that $\eta$ is an epimorphism with kernel $U \cap \imath N$, and the diagram

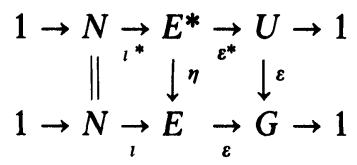

commutes and has exact rows, where $\varepsilon^{*}((u, n))=u$ for $(u, n) \in E^{*}$, $\imath^{*}(n)=(1, n)$.

Let an embedding problem $P=P(K / k, \Sigma, \gamma)$ be given and let $U$ be as above. We define the embedding problem $P_{1}=P\left(K / k, \Sigma_{1}, \gamma\right)$ where $\Sigma_{1}$ is the sequence $1 \rightarrow l^{-1}(U \cap \imath N) \rightarrow_{\imath} U \rightarrow_{\varepsilon} G \rightarrow 1$. Suppose $P_{1}$ has a solution $\left(L_{1}, \beta_{1}\right)$. We then define the embedding problem

$$
P_{2}=P\left(L_{1} / k, \Sigma_{2}, \beta_{1}\right)
$$

where $\Sigma_{2}$ is $1 \rightarrow N \rightarrow_{t^{*}} E^{*} \rightarrow_{\varepsilon^{*}} U \rightarrow 1$. Suppose $P_{2}$ has a solution $\left(L_{2}, \beta_{2}\right)$.

AMS 1970 subject classifications. Primary 12A55, 12B15, 20G40.

1 The author wishes to thank Professor Hans Zassenhaus, under whose supervision this research, which is part of the author's doctoral thesis, was done. The complete version of this paper will appear in J. Number Theory. 
Let $L$ be the fixed field of the kernel of $\eta \beta_{2}: \bar{E}_{2} \rightarrow E$, let $\bar{E}=\mathrm{Gal}(L / k)$, $\bar{N}=\mathrm{Gal}(L / K)$, and let $\beta$ be defined by means of the commutative diagram

$$
\begin{aligned}
& \bar{E}_{2} \stackrel{\beta_{2}}{\rightarrow} E^{*} \\
& \downarrow^{\text {Res }} \downarrow^{\eta} \\
& \bar{E} \underset{\beta}{\rightarrow} E
\end{aligned}
$$

One verifies that $(L, \beta)$ is a solution to $P$, hence

THEOREM 1. If the embedding problems $P_{1}, P_{2}$ have successive solutions, then so does $P$.

A GROUP-THEORETIC LEMMA. Let $E$ be a finite group, $N$ a normal subgroup. Then there exists a subgroup $U$ of $E$ such that $U N=E$ and $U \cap N$ is nilpotent, and such that if $E / N$ is nilpotent, then $U$ is nilpotent.

Indeed, one shows that a minimal subgroup $U$ such that $U N=E$ does the trick. Theorem 1 and the above lemma yield

THEOREM 2. Any embedding problem $P=P(K / k, \Sigma, \gamma)$ can be reduced to the succession of two embedding problems

$$
P_{1}=P\left(K_{1} / k_{1}, \Sigma_{1}, \gamma_{1}\right), \quad P_{2}=P\left(K_{2} / k_{2}, \Sigma_{2}, \gamma_{2}\right)
$$

(where $\Sigma_{i}$ is the exact sequence $1 \rightarrow N_{i} \rightarrow_{t_{i}} E_{i} \rightarrow_{e_{i}} G_{i} \rightarrow 1$ ), in which

in $P_{1}: \quad N_{1}$ is nilpotent;

if $G_{1}$ is solvable, then $E_{1}$ is solvable;

if $G_{1}$ is nilpotent, then $E_{1}$ is nilpotent;

in $P_{2}$ : $\Sigma_{2}$ splits.

2. On Ikeda's theorem. Theorem 1 furnishes a proof of the following theorem of Ikeda ([1], [2]): let $k$ be a number field, $P=P(K / k, \Sigma, \gamma)$ an embedding problem with $N$ abelian. If $P$ has an improper solution, then $P$ has a proper solution.

Let $\left(L_{1}, \beta_{1}\right)$ be an improper solution to $P$. Setting $U=\beta_{1}(\bar{E})$, where $\bar{E}=\operatorname{Gal}(L / k)$, we have $U_{l}(N)=E$. Moreover $\left(L_{1}, \beta_{1}\right)$ is a proper solution to $P_{1}=P\left(K / k, \Sigma_{1}, \gamma\right)$, with $P_{1}$ defined as in Theorem 1. In $P_{2}$ (defined as in Theorem 1), $\Sigma_{2}$ splits and $N$ is abelian. But Scholz [3] proved in 1929 that every embedding problem $P(K / k, \Sigma, \gamma)$ with $k$ a number field, $N$ abelian, and $\Sigma$ split, has a (proper) solution. Ikeda's theorem now follows from Theorem 1.

3. Irreducible embedding problems. Let an embedding problem $P$ $=P(K / k, \Sigma, \gamma)$ be given. Suppose $H$ is a normal subgroup of $E$, $H \cap \imath N=1$. Consider the exact and commutative diagram 


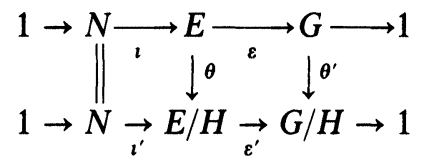

where $\theta, \theta^{\prime}$ are canonical, and $\imath^{\prime}, \varepsilon^{\prime}$ are defined so that the diagram commutes. There results a "reduced" embedding problem $P^{\prime}=P\left(K^{\prime} / k, \Sigma^{\prime}, \gamma^{\prime}\right)$ where $K^{\prime}$ is the fixed field of $\gamma^{-1} \varepsilon(H), \Sigma^{\prime}$ the bottom row of the above diagram, and $\gamma^{\prime}: \bar{G} / \gamma^{-1} \varepsilon H \rightarrow G / \varepsilon H$ is induced by $\gamma$.

THEOREM 3. $P$ has a solution if and only if $P^{\prime}$ has a solution $\left(L^{\prime}, \beta^{\prime}\right)$ such that $L^{\prime} \cap K=K^{\prime}$.

Suppose now that the center $Z(N)$ of $N$ is trivial. Set $H=Z_{E}(l N)$, the centralizer of $\imath N$ in $E$. Then $H \cap \imath N=1$ and $E^{\prime}=E / H$ is isomorphic to a subgroup of the automorphism group Aut $N$ of $N$, where the isomorphism $\eta: E^{\prime} \rightarrow$ Aut $N$ is defined by the equation $\eta\left(e^{\prime}\right)(n)=\imath^{\prime-1}\left(e^{\prime-1} \imath^{\prime}(n) e^{\prime}\right)$, $e^{\prime} \in E^{\prime}, n \in N$. Applying Theorem 3, we have

THEOREM 4. If $Z(N)=1$, then any embedding problem $P=P(K / k, \Sigma, \gamma)$ reduces to an embedding problem $P^{\prime}=P\left(K^{\prime} / k, \Sigma^{\prime}, \gamma^{\prime}\right)$, where $k \subseteq K^{\prime} \subseteq K$, where $\Sigma^{\prime}$ denotes an exact sequence $1 \rightarrow N \rightarrow E^{\prime} \rightarrow G^{\prime} \rightarrow 1$ in which $E^{\prime} \subseteq$ Aut $N$, and where the solution field is required to satisfy the condition $L^{\prime} \cap K=K^{\prime}$.

$P^{\prime}$ is called an irreducible embedding problem.

REMARK. Schreier's conjecture states that the outer automorphism group of a finite simple group is solvable. If $P=P(K / k, \Sigma, \gamma)$ is an embedding problem with $N$ simple (nonabelian), Theorem 3 reduces $P$ to the case $G$ solvable, provided Schreier's conjecture is correct. But then Theorem 2 reduces $P$ to the pair $P_{1}, P_{2}$ in which $E_{1}$ is solvable and $\Sigma_{2}$ splits. Of course it is required that $L_{1}, L_{2}$ satisfy the appropriate disjointness condition of Theorem 4 .

4. Localizability of an embedding problem. Let $k$ be a number field, $K / k$ a finite Galois extension. Let $\mathfrak{g}$ be a prime of $k$, and assume $k$ is contained in the completion $k_{\mathfrak{g}}$ of $k$ at $\mathfrak{g}$, and that $k_{\mathfrak{g}}$ is contained in an algebraic closure $\tilde{k}_{\mathrm{g}}$ of $k_{\mathrm{g}}$. Let $\sigma_{\mathrm{K}}$ be an embedding of $K$ into $\tilde{k}_{\mathrm{g}}$ extending the inclusion map of $k$ into $\tilde{k}_{\mathrm{g}}$, and inducing a prime $\mathfrak{p}$ of $K . \sigma_{K}$ induces an isomorphism $\sigma_{K}^{*}: G\left(K_{\mathfrak{p}} / k_{\mathrm{g}}\right) \rightarrow \bar{G}(\mathfrak{p})$, where $K_{\mathfrak{p}}=k_{\mathrm{g}} \cdot \sigma_{K}(K), \bar{G}=\mathrm{Gal}(K / k)$, and $\bar{G}(\mathfrak{p})$ is the decomposition group of $\mathfrak{p}$ in $\bar{G}$. $\sigma_{K}^{*}$ is given by $\sigma_{K}^{*}(\theta)(x)$ $=\sigma_{K}^{-1} \theta \sigma_{K}(x), \theta \in G\left(K_{\mathfrak{p}} / k_{\mathfrak{g}}\right), x \in K$.

Let an embedding problem $P=P(K / k, \Sigma, \gamma)$ be given. There is induced a local embedding problem $P_{\mathfrak{p}}=P\left(K_{\mathfrak{p}} / k_{\mathfrak{g}}, \Sigma_{\mathfrak{p}}, \gamma_{\mathfrak{p}}\right)$, where $\Sigma_{\mathfrak{p}}$ is the exact sequence $1 \rightarrow N \rightarrow{ }_{\imath} E_{\mathfrak{p}} \rightarrow_{\varepsilon_{\mathfrak{p}}} G_{\mathfrak{p}} \rightarrow 1$, in which $G_{\mathfrak{p}}=\gamma(\bar{G}(\mathfrak{p})), E_{\mathfrak{p}}=\varepsilon_{\mathfrak{p}}^{-1}\left(G_{\mathfrak{p}}\right)$, $\varepsilon_{\mathfrak{p}}=\left.\varepsilon\right|_{E_{\mathfrak{p}}}$, and $\gamma_{\mathfrak{p}}=\gamma \sigma_{K}^{*}$. 
Suppose $(L, \beta)$ is a solution to $P$. Let $\sigma_{L}$ be an extension of $\sigma_{K}$ to $L$, $q$ the prime of $L$ induced by $\sigma_{L}$, and let $L_{\mathrm{q}}=k_{\mathrm{g}} \sigma_{L}(L)$. Then $\left(L_{\mathrm{q}}, \beta_{\mathrm{q}}\right)$ is an improper solution to $P_{p}$, where $\beta_{\mathrm{q}}=\beta \sigma_{L}^{*}, \sigma_{L}^{*}$ defined analogous to $\sigma_{K}^{*}$. By the localization hypothesis $\mathscr{L}(P)$ we mean the following: let an embedding problem $P=P(K / k, \Sigma, \gamma)$ be given, $k$ a number field. Let $S$ be a finite set of primes of $k$, and let there be associated with each $g \in S$ a prime $\mathfrak{p}$ of $K$ dividing $\mathfrak{g}$ together with an embedding $\sigma_{K}$ defined as above. Let $P_{\mathrm{p}}$ denote the local embedding problem induced by $P$ for each $\mathrm{g} \in S$. Suppose that for each $\mathfrak{g} \in S$, the set $\mathscr{S}_{\mathfrak{g}}$ of improper solutions to $P_{\mathfrak{p}}$ is not empty. Now let there be chosen from each $\mathscr{S}_{\mathfrak{g}}$ an improper solution $\left(L^{p}, \beta^{p}\right)$. Then, there exists a finite Galois extension $L / k, L \supset K$, such that $\operatorname{Gal}(L / K) \cong N$, and the following hold: (i) for each $g \in S$, there exists an extension $\sigma_{L}$ of $\sigma_{K}$ to $L$ such that $k_{\mathrm{g}} \sigma_{L}(L)=L^{\mathfrak{p}}$, and (ii) there is an isomorphism $\alpha: \bar{N} \rightarrow N(\bar{N}=\mathrm{Gal}(L / K))$ such that for each $\mathfrak{g} \in S$, the diagram

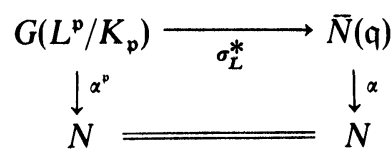

is commutative, where $\mathfrak{q}$ is induced by $\sigma_{L}, \alpha^{\mathfrak{p}}=l^{-1} \circ \beta^{\mathfrak{p}} \operatorname{Inc}_{L^{\mathfrak{p}} / K_{\mathfrak{p}}}$, and $\bar{N}(\mathfrak{q})$ is the decomposition group of $\mathfrak{q}$ in $\bar{N}$.

If $\mathscr{L}(P)$ yields a solution field $L$ to $P$, then $P$ is called localizable.

THEOREM 5. Every irreducible embedding problem in which $N=A_{n}$, the alternating group on $n$ letters, $n \neq 6, n>4$, is localizable.

EXAMPLE. Let $p_{0}, p$ be rational primes, $v$ a positive integer such that $p \mid p_{0}^{v}-1, p^{2} \nmid p_{0}^{v}-1$; for example, $p_{0}=7, p=3, v=1$. Let $q=p_{0}^{v}$, $N=P S L(p, q)$, the projective special linear group of degree $n$ over $G F(q)$, $E=P G L(p, q)$, the projective general linear group. Let $\Sigma$ be the associated canonical exact sequence. Let $k=Q(\zeta), \zeta$ a primitive $e$ th root of 1 , where $e$ is the order of $E, K=k\left(a^{1 / p}\right)$, where, by virtue of the Approximation Theorem, $a$ is chosen to have the following properties:

1. $a$ is congruent to $1 \mathrm{mod} g$ for every divisor $\mathrm{g}$ of $e$ in $k$ which is prime to $p$.

2. $a$ is congruent to $1 \operatorname{mod~}^{t_{\mathfrak{g}}}$ for every divisor $\mathrm{g}$ of $p$ in $k$, where $t_{\mathrm{g}}$ is chosen sufficiently large so that every element which is congruent to $1 \bmod ^{t^{t}}$ is the $p$ th power of an element of $k$.

3. $a$ is congruent mod $g_{0}$ to a root of unity in $k_{\mathrm{g}_{0}}$ which is not a pth power, where $g_{0}$ is any prime different from all $g$ in 1 and 2 above.

Because of the way $a$ is chosen, all the divisors of $e$ in $k$ split completely in $K$. Finally, let $\gamma$ be any isomorphism from $\bar{G}=\mathrm{Gal}(K / k)$ onto $G=E / N$. Then, the embedding problem $P=P(K / k, \Sigma, \gamma)$ is not localizable.

REMARK. The only general method known for constructing extensions 
$K$ of an arbitrary number field $k$ with arbitrary solvable Galois group $G$ is that of Safarevic [4]. All the extensions $K / k$ that he constructs have the property that every prime divisor of the order of $G$ in $k$ splits completely in $K$. The example above shows that Safarevic's method, together with the localization hypothesis, is not sufficient to solve the inverse problem of Galois Theory.

\section{REFERENCES}

1. M. Ikeda, Zur Existenz eigentlicher galoisscher Körper beim Einbettungsproblem für galoissche Algebren, Abh. Math. Sem. Univ. Hamburg 24 (1960), 126-131. MR 22 \# 12103.

2. K. Hoechsmann, Zum Einbettungsproblem, J. Reine Angew. Math. 229 (1968), 81-106. MR 39 \# 5507.

3. A. Scholz, Über die Bildung algebraischer Zahlkorper mit auflosbarer Galoisscher Gruppe, Math Z. 30 (1929), 332-356.

4. I. R. Safarevic, On the construction of fields with a given Galois group of order, Izv. Akad. Nauk SSSR Ser. Mat. 18 (1954), 261-296; English transl., Amer. Math. Soc. Transl. (2) 4 (1956), 107-142. MR 16, 571.

Department of Mathematics, Adelphi University, Garden City, New York 11530 